\title{
Gold Nanoparticles Functionalized by a Luminescent Electroactive Complex : Investigation of Photophysical and Electrofluorochromic Properties
}

\author{
L. Guerret ${ }^{1}$, J.F. Audibert ${ }^{1}$, A. Debarre ${ }^{1,2}$, M. Lepeltier ${ }^{3}$, P. Haghi-Ahstiani ${ }^{4}$, G. Dubacheva ${ }^{1}$, F. \\ Miomandre ${ }^{1^{*}}$
}

\section{Supplementary information}

Figure captions :

Figure S1: Spectral features of the excitation filter (blue line), dichroic mirror (green line) and emission filter (red line) used in the EF study.

Figure S2 : Absorption spectra of the Ir-Au NP colloidal suspension in DCB : right after synthesis (red full line); after 12 days (green dashed line); after 20 days (dashed blue line).

Figure S3 : Experimental correlation profiles of sample S3 (Red dots) and fit according to Eqns.(1) and (3), respectively (blue lines); on top residue of the fit; excitation wavelength and power are equal to $720 \mathrm{~nm}$ and $6 \mathrm{~mW}$, respectively. Concentration of $\mathrm{Ir}=10^{-7} \mathrm{M}$ in the mixing.

Figure S4 : Modulation of luminescence of Au-Ir NP (blue trace) vs. time upon potential steps (red trace) between 0 and $1.5 \mathrm{~V}$

\footnotetext{
*Corresponding author : mioman@ppsm.ens-cachan.fr

${ }_{1}^{1}$ PPSM, CNRS, ENS Cachan - Université Paris-Saclay, 61 Avenue Président Wilson, 94235 CACHAN, France

${ }^{2}$ LAC, CNRS, Université Paris 11, ENS Cachan, Université Paris-Saclay, Campus d'Orsay, Bât 405, 91405 ORSAY, France

${ }^{3}$ ILV - CNRS, Université Versailles Saint Quentin, 45 Avenue des Etats-Unis, 78035 VERSAILLES, France

${ }^{4}$ LSSMMAT -CNRS, Ecole CentraleSupélec- Grande Voie des Vignes, 92290 CHATENAY-MALABRY
} 
Figure S1

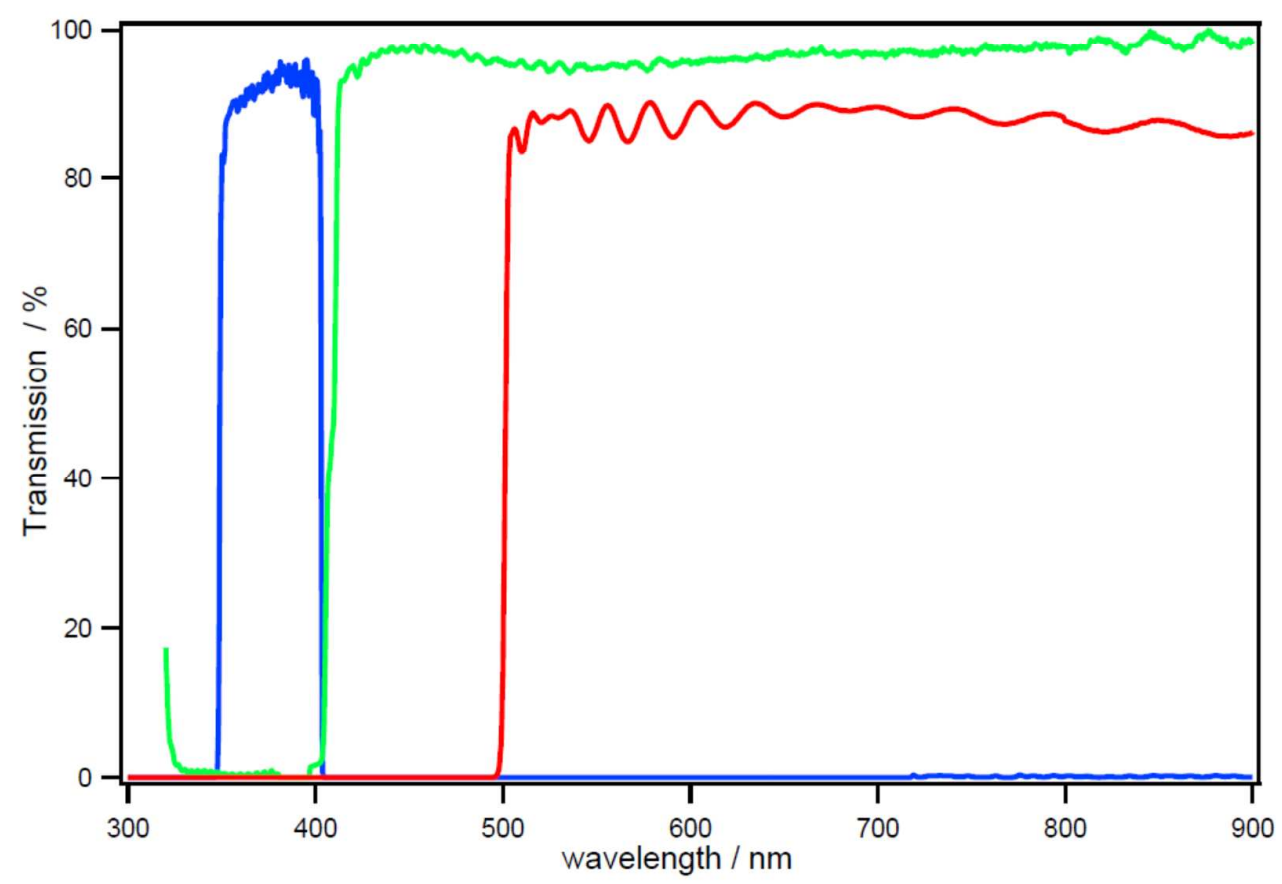


Figure S2




Figure S3




Figure S4

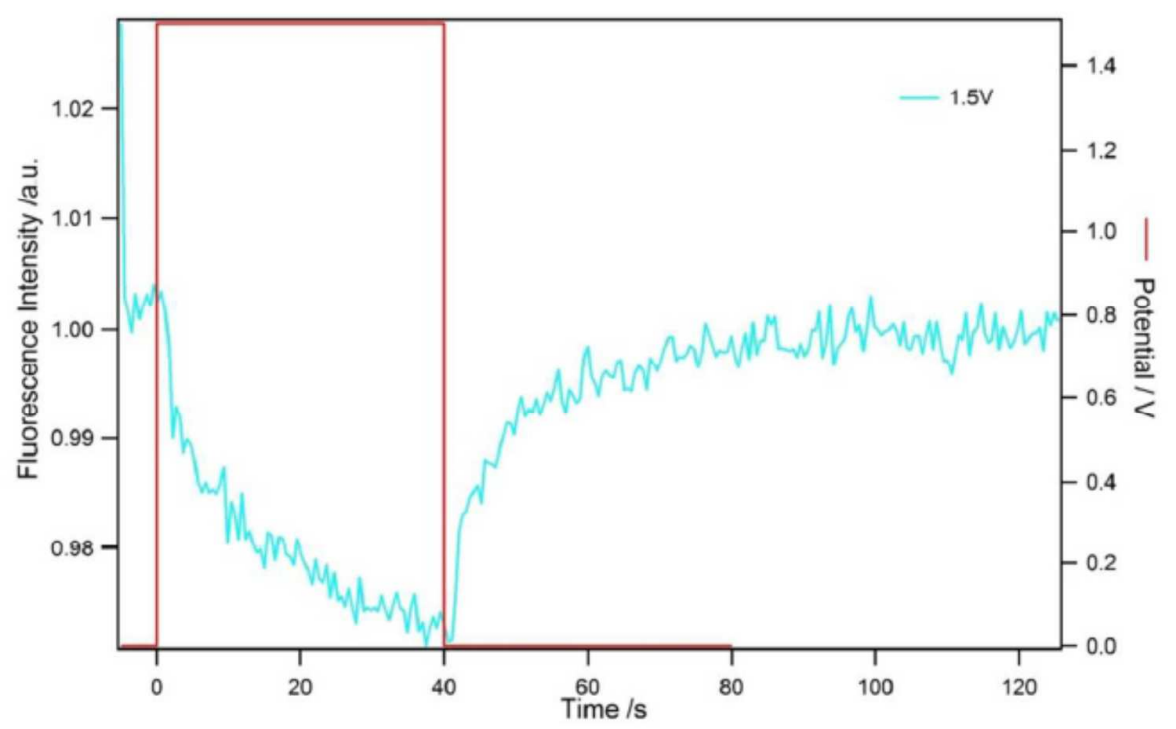

